\title{
MULTI-SCALE METHOD FOR THE CRACK PROBLEM IN MICROSTRUCTURAL MATERIALS
}

\author{
R. H. W. HOPPE ${ }^{1}$ AND S. I. PETROVA ${ }^{2}$
}

\begin{abstract}
The paper deals with the numerical computation of a crack problem posed on microstructural heterogeneous materials containing multiple phases in the microstructure. The failure of such materials is a natural multi-scale effect since cracks typically nucleate in regions of defects on the microscopic scale. The modeling strategy for solving the crack problem concerns simultaneously the macroscopic and microscopic models. Our approach is based on an efficient combination of the homogenization technique and the mesh superposition method ( $s$-version of the finite element method). The homogenized model relies on a double-scale asymptotic expansion of the displacement field. The mesh superposition method uses two independent (global and local) finite element meshes and the concept of superposing the local mesh arbitrarily on the global continuous mesh. The crack is treated by the local mesh and the homogenized material model is considered on the global mesh. Numerical experiments for problems on biomorphic microcellular ceramic templates with porous microstructures of different materials constituents are presented.
\end{abstract}

2000 Mathematics Subject Classification: 65M60, 74B05, 74Q05, 74Q15, 74R10.

Keywords: homogenization, elasticity, mesh superposition, crack, extended FEM.

\section{Introduction}

Various heterogeneous (porous and composite) materials with multiple scales and multiple phases in the microstructure have been produced in recent years. Due to the advanced manufacturing technologies and scientific computations they have found a lot of applications and wide use in the automotive and aerospace industries, in chemistry, and in medicine as implants for replacing traditionally used material structures.

The asymptotic homogenization theory (cf., e.g., $[2,5,18,29]$ ) has been successfully used in the last three decades for solving multi-scale problems on computational regions occupied by heterogeneous microstructural materials. In general, the microscopic and macroscopic models are considered simultaneously supposing a strong scale separation, i.e., a large gap in the length scale between the macroscopic component and the microstructure. In practical applications the microscopic length scales are orders of magnitude smaller than the physical macroscopic length scale. The main assumption in the homogenization approach is that the

\footnotetext{
${ }^{1}$ Institute of Mathematics, University of Augsburg, D-86159 Augsburg, Germany; Department of Mathematics, University of Houston, Houston, TX 77204-3008, USA.

${ }^{2}$ Department of Mathematics, University of Applied Sciences, D-33609 Bielefeld, Germany; Institute for Parallel Processing, Bulgarian Academy of Sciences, 1113 Sofia, Bulgaria. E-mail: svetozara.petrova@fhbielefeld.de
} 
original heterogeneous material workpiece is composed of periodically distributed microstructures of various constituents (or different phases). For convenience, the microstructures are chosen as microscopic unit cells. The microscopic scale is typically characterized by the inner heterogeneity, and the macroscopic scale concerns the global dimension of the structure. A double-scale asymptotic expansion for the displacement field and a homogenization procedure by taking a zero limit of the scale ratio are applied to come up with computationally feasible macromodels. The asymptotic homogenization theory is nowadays used as a powerful tool in the materials science and the computational mechanics for optimal design of composite structures $[1,4,12]$.

The homogenization theory based on the averaging procedures and effective constitutive laws can be treated as a part of the heterogeneous multi-scale method, recently presented in [8] as a general methodology for the computation of problems with multi-scales and multiphysics. The method relies on the analysis and efficient coupling of different physical models (for instance, macroscopic and microscopic ones) on different scales and different meshes. Several examples and a large variety of real-life multi-scale problems are discussed in detail in the latter paper.

Mechanical failure due to the initiation and propagation of cracks in places of high pore density in the microstructure is considered in this study. In the vicinity of microcracks the microscopic periodicity in the macromodel is lost. The fracture of the materials causes a local heterogeneity and discontinuities in the crack region for which the asymptotic homogenization theory cannot be applied in a standard fashion. In this case, the model relies on a failure zone model in the crack vicinity incorporating microstructural information on the pore growth processes combined with the homogenized model of the failure zone. The main difficulty in the computational simulation of discrete fracture models is the proper measurement of microscopic variables and characteristic quantities which requires a substantial amount of data from experimental investigations. Moreover, the spatial discretization has to be correctly adjusted to the changing topology of the domain which typically appears in the failure zone. Hence, special methods have to be developed to implicitly model the internal discontinuities. Previous studies on multi-scale modeling of crack and damage propagation in composite materials can be found in [21,27].

The asymptotic homogenization theory together with the mesh superposition method ( $s$-version of the Finite Element Method), first introduced in [9] and developed later on in $[11,13]$, is used for the multi-scale analysis of our microstructural materials. The crack problem is modeled by the global-local approach in which different regions of the problem domain are covered by different discrete meshes. For early applications of the superposition method to problems with mechanical failures we refer the reader to [28,30]. Recently, an enhanced mesh superposition method involving homogenization has been applied in [31] for porous ceramics with microscopic cracks. In [22], a hybrid method is considered in which the superposition approach is applied only for the crack tips and the rest of the crack is treated by the eXtended Finite Element Method (XFEM). The latter method has been recently proposed (see the pioneering work [24]) as a numerical technique which facilitates crack propagation simulations to be conducted without explicitly meshing the crack surfaces or remeshing as the crack grows.

The remaining of this paper is organized as follows. The multi-scale method for the crack problem in heterogeneous materials is formulated in Section 2. The method is based on the combination of the asymptotic homogenization theory and the mesh superposition method. The homogenized material model is considered in the global mesh. However, it cannot be 
applied in the local discontinuous region since the microscopic periodicity assumption fails in the vicinity of cracks. The heterogenous microstructures are modeled using the material properties of the microstructural constituents. Within the framework of the $s$-version of the FEM, the local mesh generated near the crack is arbitrarily superimposed onto the global mesh. The homogenized macroscopic model is presented in Section 3. We assume that both macro- and microscales are well separated. The homogenization approach requires that: 1) the elasticity partial differential equation be solved in the unit microstructure in order to find the periodic displacements needed to compute the effective (homogenized) coefficients and 2) the macroscopic homogenized problem on the whole domain be solved. The effective macroscopic material properties are predicted from the properties of the constituents assuming a periodical distribution of the microstructures. The generation of global and local meshes and the multi-scale solution algorithm are discussed in Section 4. Section 5 gives a brief description of the XFEM in which the finite element space is enriched by adding special functions to the approximation through the notion of partition of unity [23]. Some numerical experiments for biomorphic cellular ceramics with porous microstructures produced from natural wood are presented in [6]. More computational results are given in the last section.

Everywhere throughout this study bold-face symbols indicate variables of tensorial character. The Einstein convention of summation over repeated indices has been adopted, so that we have, for instance, $(\boldsymbol{\sigma} \boldsymbol{n})_{i}=\sigma_{i j} n_{j}$ and $\sigma_{i j}=E_{i j k l} e_{k l}$.

\section{Multi-scale method for the crack problem}

2.1. Cracking failure. Heterogeneous microstructural materials with porous microstructures are considered in this paper. Bending effects such as initiation and propagation of micro- and macrocracks may activate various failure mechanisms in these materials. Failure typically occurs in the case of bending loads in places of high pore density and can be viewed as a material-dependent local phenomenon in regions parallel to the pore channel orientation. For the simulation of macrocrack initiation and propagation, microscopic effects such as the nucleation and growth of microcracks in ductile materials have to be incorporated (see Fig. 2.1). For these materials a relatively large plastic zone exists at the macrocrack front. Microcracks can appear within this plastic zone. In the vicinity of microcracks the material becomes softer and tougher due to the increasing pore size. This has the effect of shielding the macrocrack from the applied load.

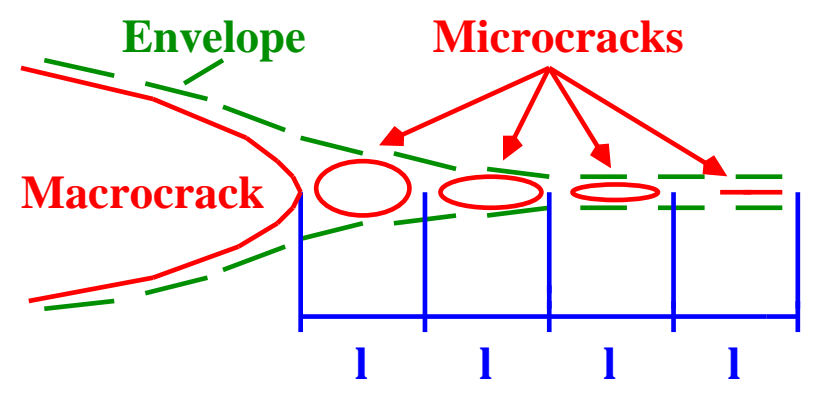

Fig. 2.1. Evolution of microcracks near the crack tip (ductile materials)

In Section 6, we consider brittle ceramic materials without plastic zones. Note that the approach proposed in the paper is not restricted to the above materials but can also be applied to ductile materials. In general, methods of fracture mechanics can be used for a 
finite amount of microscopic discrete defects, while methods of damage mechanics hold for materials of wide-spread distributed defects. Various failure models and more sophisticated damage models are discussed in [19].

In what follows, we emphasize the necessity of multi-scale algorithms and global-local analysis to describe the local microstructural phenomena accurately.

2.2. Global-local approach for heterogeneous materials. Consider a domain $\Omega \subset \mathcal{R}^{d}, d=2,3$, occupied by a heterogeneous material with microstructures of periodically distributed constituents. Suppose that the boundary of $\Omega$ denoted by $\Gamma$ consists of a prescribed displacement boundary $\Gamma_{D}$ (meas $\left.\Gamma_{D}>0\right)$ and a prescribed traction boundary $\Gamma_{T}$, such that $\Gamma=\Gamma_{D} \cup \Gamma_{T}, \Gamma_{D} \cap \Gamma_{T}=\varnothing$, as shown in Fig. 2.2.

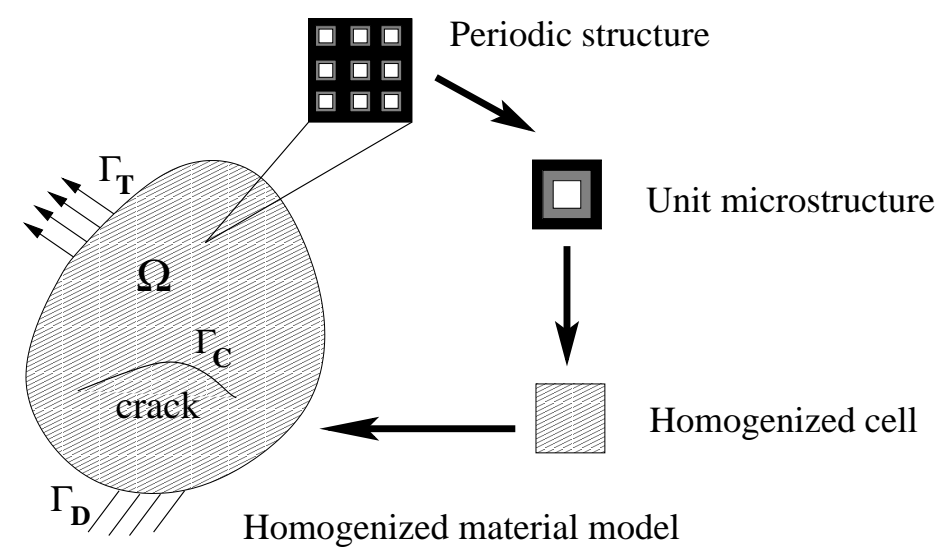

Fig. 2.2. Crack in the macroscopic homogenized material model

Assume that the material macrostructure is composed of periodically repeated cells. The unit microstructure consists of different material constituents and a pore. Both the macroscopic and microscopic scales are well separated, i.e., the size of the microstructure in the heterogeneous material is much smaller than those of the macroscopic component. The asymptotic homogenization theory is applied to find the effective (homogenized) properties of the material and to derive the homogenized macroscopic model. Details are given in Section 3. The main idea for the homogenization of a heterogeneous material with a periodical distribution of microstructures is illustrated in Fig. 2.2.

We allow the domain $\Omega$ to contain discontinuities and consider the crack problem with a crack $\Gamma_{C}$ (see Fig. 2.2). The crack is a multi-scale effect which typically appears in regions with microstructures of increasing porosity. This effect is shown on the microscopic level in Fig. 2.3. The periodicity fails for those microstructures cut by the crack. Therefore, a new finite element analysis has to incorporate microstructural information about the nucleation and growth of micropores. This has to be done in such a way that the computational results do not depend on the granularity of the underlying finite element mesh (mesh independence).

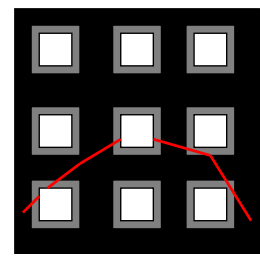

Fig. 2.3. Local heterogeneity near the microcrack

We rely on the mesh superposition method (known as the $s$-version of the FEM), introduced in [9] and developed later in $[11,13]$. The method is based on a finite element 
approximation using two independent meshes: a global mesh for the entire domain (also called in the literature the background mesh) and a local mesh in the critical region near the crack (also called the patch mesh). The local mesh is arbitrarily superimposed on the global mesh without taking care of the matching between nodes in both meshes.

We consider first the following governing equations in the domain $\Omega$ :

$$
\begin{gathered}
-\operatorname{div} \boldsymbol{\sigma}(\boldsymbol{u})=\boldsymbol{b} \quad \text { in } \Omega, \\
\boldsymbol{u}=\boldsymbol{g} \quad \text { on } \Gamma_{D}, \\
\boldsymbol{\sigma} \boldsymbol{n}=\boldsymbol{t} \quad \text { on } \Gamma_{T},
\end{gathered}
$$

where $\boldsymbol{\sigma}$ is the second order symmetric stress tensor, $\boldsymbol{u}$ is the unknown displacement field, $\boldsymbol{b}$ is the body force, $\boldsymbol{g}$ is the prescribed displacement on $\Gamma_{D}, \boldsymbol{t}$ is the prescribed traction on $\Gamma_{T}$, and $\boldsymbol{n}$ is the unit normal to the boundary $\Gamma_{T}$. A traction-free surface $\boldsymbol{\sigma} \boldsymbol{n}=\mathbf{0}$ is assumed on the crack $\Gamma_{C}$.

In the case of small strains and displacements, the second-order strain tensor $\boldsymbol{e}$ is

$$
\boldsymbol{e}=\boldsymbol{e}(\boldsymbol{u})=\left(\nabla \boldsymbol{u}+(\nabla \boldsymbol{u})^{T}\right) / 2,
$$

where $\nabla \boldsymbol{u}$ is the gradient operator. If linear elasticity is assumed, then the constitutive relation is given by the linearized Hooke law

$$
\sigma=E \boldsymbol{e}
$$

where $\boldsymbol{E}$ is the forth-order elasticity tensor depending on material constants like Young's modulus and Poisson's ratio.

For convenience, further in this section, due to the symmetry of the stress and strain tensors, we consider the strain in the vector form $\boldsymbol{e}=\boldsymbol{B} \boldsymbol{u}$, where $\boldsymbol{B}$ is the gradient operator (also called the strain-displacement matrix). The weak form of the governing equation (2.1) then reads: Find $\boldsymbol{u} \in U$ such that

$$
\int_{\Omega} \boldsymbol{e}^{T}(\boldsymbol{v}) \boldsymbol{\sigma}(\boldsymbol{u}) d \Omega=\int_{\Omega} \boldsymbol{v}^{T} \boldsymbol{b} d \Omega+\int_{\Gamma_{T}} \boldsymbol{v}^{T} \boldsymbol{t} d \Gamma, \quad \forall \boldsymbol{v} \in U_{0},
$$

where the set of admissible displacement fields is defined by

$$
U=\left\{\boldsymbol{v} \mid \boldsymbol{v} \in V, \boldsymbol{v}=\boldsymbol{g} \text { on } \Gamma_{D}, \boldsymbol{v} \text { discontinuous on } \Gamma_{C}\right\}
$$

and the test function space is defined by

$$
U_{0}=\left\{\boldsymbol{v} \mid \boldsymbol{v} \in V, \boldsymbol{v}=\mathbf{0} \text { on } \Gamma_{D}, \boldsymbol{v} \text { discontinuous on } \Gamma_{C}\right\} .
$$

Here, the space $V$ is related to the regularity of the solution in $\Omega$ and allows for discontinuous functions across the crack. At each point $\boldsymbol{x} \in \Omega$ we consider a finite element discretization of (2.6) with a basis taken from the test function space $U_{0}$ and nodal shape functions $\boldsymbol{N}(\boldsymbol{x})$ constructed by the Galerkin method.

We denote the local critical region on which the local mesh is superimposed by $\Omega^{L}, \Omega^{L} \subset$ $\Omega$, a subset of $\Omega$, containing the crack. Let $\Omega^{G}=\Omega \backslash \Omega^{L}$ be the rest of the domain excluding discontinuities. The domains $\Omega$ and $\Omega^{L}$ are discretized independently by separate sets of finite elements $\Omega_{e}^{G}$ and $\Omega_{e}^{L}$, such that $\bigcup \Omega_{e}^{G}=\Omega$ and $\bigcup \Omega_{e}^{L}=\Omega^{L}$. Here, the superscript $G$ 
relates to the global (underlying) mesh and $L$ to the local (superimposed) mesh. $\Gamma^{G L}$ is the boundary between the two meshes excluding external boundaries, i.e., $\Gamma \cap \Gamma^{G L}=\varnothing$.

Let $\boldsymbol{u}^{G}$ be the global displacement field defined in $\Omega$ and $\boldsymbol{u}^{L}$ be the local displacement field defined in the local region $\Omega^{L}$. Note that the superimposed field $\boldsymbol{u}^{L}$ is in general discontinuous due to the discontinuity across the crack faces. The total displacement field $\boldsymbol{u}$ is constructed by superposition of both displacement fields on the separate meshes and can be written as follows:

$$
\boldsymbol{u}= \begin{cases}\boldsymbol{u}^{G} & \text { on } \Omega^{G}, \Gamma^{G L} \\ \boldsymbol{u}^{G}+\boldsymbol{u}^{L} & \text { on } \Omega^{L} .\end{cases}
$$

To ensure displacement compatibility between the global and local finite element meshes, we assume homogeneous boundary conditions on the boundary of the patch, i.e.,

$$
\boldsymbol{u}^{L}=\mathbf{0} \text { on } \Gamma^{G L} .
$$

Denote by $\boldsymbol{B}^{G}$ and $\boldsymbol{B}^{L}$ the strain-displacement matrices for the global and local meshes, respectively. Then, the strain can be expressed as follows:

$$
\boldsymbol{e}= \begin{cases}\boldsymbol{e}^{G}=\boldsymbol{B}^{G} \boldsymbol{u}^{G} & \text { on } \Omega^{G} \\ \boldsymbol{e}^{L}=\boldsymbol{B}^{G} \boldsymbol{u}^{G}+\boldsymbol{B}^{L} \boldsymbol{u}^{L} & \text { on } \Omega^{L} .\end{cases}
$$

Following (2.5), we get the following constitutive relations for the global and local meshes:

$$
\boldsymbol{\sigma}=\boldsymbol{E} \boldsymbol{e}= \begin{cases}\boldsymbol{\sigma}^{G}=\boldsymbol{E}^{G}\left(\boldsymbol{B}^{G} \boldsymbol{u}^{G}\right) & \text { on } \Omega^{G} \\ \boldsymbol{\sigma}^{L}=\boldsymbol{E}^{L}\left(\boldsymbol{B}^{G} \boldsymbol{u}^{G}+\boldsymbol{B}^{L} \boldsymbol{u}^{L}\right) & \text { on } \Omega^{L},\end{cases}
$$

where $\boldsymbol{E}^{G}$ and $\boldsymbol{E}^{L}$ are the elasticity tensors corresponding to the different constitutive laws. By using shape functions $\boldsymbol{N}^{G}(\boldsymbol{x})$ on the global mesh and shape functions $\boldsymbol{N}^{L}(\boldsymbol{x})$ on the local mesh (both presented in a matrix notation), one can get from the standard weak form (2.6) the following two equations:

$$
\begin{gathered}
\int_{\Omega^{G}}\left(\boldsymbol{B}^{G}(\boldsymbol{x})\right)^{T} \boldsymbol{\sigma}^{G}(\boldsymbol{u}) d \Omega=\int_{\Omega^{G}}\left(\boldsymbol{N}^{G}(\boldsymbol{x})\right)^{T} \boldsymbol{b} d \Omega+\int_{\Gamma_{T}^{G}}\left(\boldsymbol{N}^{G}(\boldsymbol{x})\right)^{T} \boldsymbol{t} d \Gamma, \\
\int_{\Omega^{L}}\left(\boldsymbol{B}^{L}(\boldsymbol{x})\right)^{T} \boldsymbol{\sigma}^{L}(\boldsymbol{u}) d \Omega=\int_{\Omega^{L}}\left(\boldsymbol{N}^{L}(\boldsymbol{x})\right)^{T} \boldsymbol{b} d \Omega+\int_{\Gamma_{T}^{L}}\left(\boldsymbol{N}^{L}(\boldsymbol{x})\right)^{T} \boldsymbol{t} d \Gamma,
\end{gathered}
$$

where $\Gamma_{T}^{G}=\Gamma_{T} \cap \Omega^{G}$ and $\Gamma_{T}^{L}=\Gamma_{T} \cap \Omega^{L}$. Substituting (2.9)-(2.12) into equations (2.13), (2.14), we obtain the following discrete system:

$$
\left[\begin{array}{ll}
\boldsymbol{K}^{G} & \boldsymbol{K}^{G L} \\
\left(\boldsymbol{K}^{G L}\right)^{T} & \boldsymbol{K}^{L}
\end{array}\right]\left\{\begin{array}{l}
\boldsymbol{u}^{G} \\
\boldsymbol{u}^{L}
\end{array}\right\}=\left\{\begin{array}{l}
\boldsymbol{f}^{G} \\
\boldsymbol{f}^{L}
\end{array}\right\}
$$

where $\boldsymbol{K}^{G}$ and $\boldsymbol{K}^{L}$ are the stiffness matrices corresponding to the global and local meshes, respectively, and $\boldsymbol{K}^{G L}$ is the matrix corresponding to the interaction between the two meshes

$$
\boldsymbol{K}^{G}=\int_{\Omega^{G}}\left(\boldsymbol{B}^{G}(\boldsymbol{x})\right)^{T} \boldsymbol{E}^{G} \boldsymbol{B}^{G}(\boldsymbol{x}) d \Omega+\int_{\Omega^{L}}\left(\boldsymbol{B}^{G}(\boldsymbol{x})\right)^{T} \boldsymbol{E}^{L} \boldsymbol{B}^{G}(\boldsymbol{x}) d \Omega,
$$




$$
\begin{gathered}
\boldsymbol{K}^{G L}=\int_{\Omega^{L}}\left(\boldsymbol{B}^{G}(\boldsymbol{x})\right)^{T} \boldsymbol{E}^{L} \boldsymbol{B}^{L}(\boldsymbol{x}) d \Omega, \\
\boldsymbol{K}^{L}=\int_{\Omega^{L}}\left(\boldsymbol{B}^{L}(\boldsymbol{x})\right)^{T} \boldsymbol{E}^{L} \boldsymbol{B}^{L}(\boldsymbol{x}) d \Omega .
\end{gathered}
$$

The force vectors $\boldsymbol{f}^{G}$ and $\boldsymbol{f}^{L}$ are computed from the right-hand sides of (2.13) and (2.14) as

$$
\begin{aligned}
\boldsymbol{f}^{G} & =\int_{\Omega^{G}}\left(\boldsymbol{N}^{G}(\boldsymbol{x})\right)^{T} \boldsymbol{b} d \Omega+\int_{\Gamma_{T}^{G}}\left(\boldsymbol{N}^{G}(\boldsymbol{x})\right)^{T} \boldsymbol{t} d \Gamma \\
\boldsymbol{f}^{L} & =\int_{\Omega^{L}}\left(\boldsymbol{N}^{L}(\boldsymbol{x})\right)^{T} \boldsymbol{b} d \Omega+\int_{\Gamma_{T}^{L}}\left(\boldsymbol{N}^{L}(\boldsymbol{x})\right)^{T} \boldsymbol{t} d \Gamma .
\end{aligned}
$$

Note that $\boldsymbol{N}^{G}(\boldsymbol{x})$ stand for the shape functions corresponding to the finite elements in the global mesh on which a continuous displacement field $\boldsymbol{u}^{G}$ is considered. Furthermore, $\boldsymbol{N}^{L}(\boldsymbol{x})$ denote the discontinuous shape functions of the elements chosen on the local domain to model the crack. The elements of the global and local meshes should not coincide. The cracked mesh is superimposed on the continuous mesh in $\Omega^{L}$ by using the $s$-method. The main difficulty of this method is the numerical integration based on Gauss quadratures when solving system (2.15). An attractive approach has been proposed recently in [22] where only the near-tip crack fields are modeled on a superimposed patch (overlaid mesh) and the rest of the crack is treated within the framework of the XFEM by introducing additional discontinuous enrichment functions for elements completely cut by the crack (see [24]).

\section{Homogenized computational model}

In this section, we briefly explain the derivation of the homogenized computational model on the macroscale by using the asymptotic homogenization theory (see, cf., [2, 5, 18, 29]). The homogenized model for our original heterogeneous material occupying the domain $\Omega$, $\Omega \subset \mathcal{R}^{d}, d=2,3$, is illustrated in Fig. 2.2. The main idea of the homogenization is to replace the heterogeneous material by an equivalent homogenized material, extracting information for the material properties of various microstructural constituents. To couple properly the micro- and macroscales, a representative volume element (RVE) or a unit microstructure is considered.

We suppose a periodical distribution of microcells of different phases and a pore inside the unit microstructure (see Fig. 2.2). Homogenization is possible if the macro- and microscales are well separated, i.e., we assume that the periodic cells in the macrostructure are infinitely many but infinitely small and repeated periodically through the medium. We introduce two space variables $\boldsymbol{x}$ (macroscopic variable) and $\boldsymbol{y}$ (microscopic variable) and denote by $\varepsilon$, $\boldsymbol{y}=\boldsymbol{x} / \varepsilon, \varepsilon \ll 1$, the scale parameter (dimensionless number) which, in fact, represents the periodicity under the assumption that $\varepsilon$ is very small with respect to the size of $\Omega$, i.e., there exists a large scale gap between the microstructure and the macroscopic component.

The parameter $\varepsilon$ allows us to define the macrofunctions in terms of the microstructural behavior and vice versa. Thus, for any state function $f(\boldsymbol{y}):=f(\boldsymbol{x} / \varepsilon)$, one can compute the 
spatial derivatives by using the following differentiation rule:

$$
\frac{d}{d \boldsymbol{x}} f\left(\boldsymbol{x}, \frac{\boldsymbol{x}}{\varepsilon}\right)=\frac{\partial f(\boldsymbol{x}, \boldsymbol{y})}{\partial \boldsymbol{x}}+\varepsilon^{-1} \frac{\partial f(\boldsymbol{x}, \boldsymbol{y})}{\partial \boldsymbol{y}} .
$$

Denote by $Y=[0,1]^{d}, d=2,3$, the unit microstructure and consider the elasticity equation

$$
-\operatorname{div} \boldsymbol{\sigma}(\boldsymbol{u})=\mathbf{F} \quad \text { in } Y
$$

with a load vector $\mathbf{F}$. Here, $\boldsymbol{\sigma}$ is the microscopic symmetric stress, $\boldsymbol{u} \in \boldsymbol{H}^{1}(Y)$, is the corresponding displacement at point $\boldsymbol{y} \in Y$, and $\boldsymbol{e}$ is the microscopic symmetric strain with components

$$
e_{i j}(\boldsymbol{u}(\boldsymbol{y}))=\frac{1}{2}\left(\frac{\partial u_{i}(\boldsymbol{y})}{\partial y_{j}}+\frac{\partial u_{j}(\boldsymbol{y})}{\partial y_{i}}\right) .
$$

Problem (3.1) is subject to periodic boundary conditions on the outer part of $\partial Y$, Neumann boundary conditions around the pore, and continuity conditions $[\boldsymbol{u}]=0$ and $[\boldsymbol{\sigma} \overline{\boldsymbol{n}}]=\mathbf{0}$ on the interfaces between different phases (see Fig. 6.2). The symbol [] denotes the jump of the function across the corresponding interface with a normal vector $\overline{\boldsymbol{n}}$ (cf., e.g., [2]).

Assuming linearly elastic constituents, the unit microstructure is governed by the Hooke law $\boldsymbol{\sigma}=\boldsymbol{E} \boldsymbol{e}$ with componentwise $(i, j, k, l=1, \ldots, d)$ constitutive relations as follows:

$$
\sigma_{i j}(\boldsymbol{u})=E_{i j k l}(\boldsymbol{y}) e_{k l}(\boldsymbol{u}(\boldsymbol{y}))
$$

The 4th order elasticity (also called plain-stress) tensor $\boldsymbol{E}(\boldsymbol{y})$ with components $E_{i j k l}(\boldsymbol{y})$ characterizes the behavior of the material at point $\boldsymbol{y}$ and depends on material constants like Young's modulus and Poisson's ratio. Note that $\boldsymbol{E}(\boldsymbol{y})$ is zero if $\boldsymbol{y}$ is located in the porous subdomain of the microstructure and coincides with the elasticity tensor of the material if $\boldsymbol{y}$ is located in the corresponding microstructural constituent. The elasticity tensor is symmetric in the following sense:

$$
E_{i j k l}=E_{j i k l}=E_{i j l k}=E_{k l i j}
$$

and satisfies the following ellipticity conditions:

$$
E_{i j k l} \chi_{i j} \chi_{k l} \geqslant c \chi_{i j}^{2}, \quad \forall \chi_{i j}=\chi_{j i}
$$

for a constant $c>0$ (cf., e.g., $[2,5,18]$ ).

Denote by $\boldsymbol{u}_{\varepsilon}(\boldsymbol{x}):=\boldsymbol{u}(\boldsymbol{x} / \varepsilon)$ the unknown macroscopic displacement vector and consider the following family of elasticity problems:

$$
-\operatorname{div} \boldsymbol{\sigma}_{\varepsilon}\left(\boldsymbol{u}_{\varepsilon}\right)=\boldsymbol{b} \text { in } \Omega,
$$

subject to a macroscopic body force $\boldsymbol{b}$ and a macroscopic surface traction $\boldsymbol{t}$ applied to the portion $\Gamma_{T} \subset \partial \Omega$. Here, $\boldsymbol{\sigma}_{\varepsilon}\left(\boldsymbol{u}_{\varepsilon}\right):=\boldsymbol{E}_{\varepsilon}(\boldsymbol{x}) \boldsymbol{e}\left(\boldsymbol{u}_{\varepsilon}(\boldsymbol{x})\right)$ is the stress tensor for $\boldsymbol{x} \in \Omega$ and $\boldsymbol{E}_{\varepsilon}(\boldsymbol{x}):=\boldsymbol{E}(\boldsymbol{x} / \varepsilon)=\boldsymbol{E}(\boldsymbol{y})$ is the piecewise constant elasticity tensor defined in $Y$. Following, for instance, [5] for the basic concepts of the homogenization method, the unknown displacement vector is expanded asymptotically as

$$
\boldsymbol{u}_{\varepsilon}(\boldsymbol{x})=\boldsymbol{u}^{(0)}(\boldsymbol{x}, \boldsymbol{y})+\varepsilon \boldsymbol{u}^{(1)}(\boldsymbol{x}, \boldsymbol{y})+\varepsilon^{2} \boldsymbol{u}^{(2)}(\boldsymbol{x}, \boldsymbol{y})+\ldots \quad \boldsymbol{y}=\boldsymbol{x} / \varepsilon,
$$


where $\boldsymbol{u}^{(i)}(\boldsymbol{x}, \boldsymbol{y}), i \geqslant 0$, are $Y$-periodic in $\boldsymbol{y}$, i.e., take equal values on opposite sides of $Y$. Consider the space $H:=\left\{\boldsymbol{u} \mid \boldsymbol{u} \in\left(H^{1}(\Omega)\right)^{d}, \boldsymbol{u}=\mathbf{0}\right.$ on $\left.\Gamma_{D}\right\}$. Under the assumptions of symmetry and ellipticity of the elasticity coefficients, it was shown in the homogenization theory that the sequence $\left\{\boldsymbol{u}_{\varepsilon}\right\}$ of solutions of (3.5) tends weakly in $H$ as $\varepsilon \rightarrow 0$ to a function $\boldsymbol{u}^{(0)}(\boldsymbol{x}) \in H$, the solution of the following macroscopic homogenized problem with a constant elasticity tensor:

$$
-\operatorname{div} \boldsymbol{\sigma}\left(\boldsymbol{u}^{(0)}\right)=\boldsymbol{b} \quad \text { in } \Omega,
$$

where $\boldsymbol{\sigma}\left(\boldsymbol{u}^{(0)}\right):=\boldsymbol{E}^{H} \boldsymbol{e}\left(\boldsymbol{u}^{(0)}(\boldsymbol{x})\right), \boldsymbol{x} \in \Omega$, and $\boldsymbol{E}^{H}$ stands for the homogenized elasticity tensor. Note that $\boldsymbol{u}^{(0)}(\boldsymbol{x})$ depends only on the macroscopic variable $\boldsymbol{x}$ and is independent of the microscopic scale $\boldsymbol{y}$. The leading term $\boldsymbol{u}^{(0)}$ in (3.6) is called a macroscopic displacement and the remaining terms $\boldsymbol{u}^{(i)}, i>0$ are considered as perturbed displacements.

The homogenization method requires finding periodic functions $\boldsymbol{\xi}^{k l}$ satisfying the following problem in a weak formulation to be solved in the microscopic unit cell:

$$
\int_{Y} E_{i j p q}(\boldsymbol{y}) \frac{\partial \xi_{p}^{k l}}{\partial y_{q}} \frac{\partial \phi_{i}}{\partial y_{j}} d Y=\int_{Y} E_{i j k l}(\boldsymbol{y}) \frac{\partial \phi_{i}}{\partial y_{j}} d Y,
$$

where $\phi \in \boldsymbol{H}^{1}(Y)$ is an arbitrary $Y$-periodic variational function. The function $\boldsymbol{\xi}^{k l}$, also referred to as the characteristic displacement, is found by solving (3.8) in $Y$ with periodic boundary conditions, i.e., assuming the same values on opposite sides of $\partial Y$. After computing $\boldsymbol{\xi}^{k l}$, one defines the homogenized coefficients by the following formulas (we refer to $[2,5,18]$ for details):

$$
E_{i j k l}^{H}=\frac{1}{|Y|} \int_{Y}\left(E_{i j k l}(\boldsymbol{y})-E_{i j p q}(\boldsymbol{y}) \frac{\partial \xi_{p}^{k l}}{\partial y_{q}}\right) d Y .
$$

Due to the symmetry conditions (3.4), the 4th order homogenized elasticity tensor $\boldsymbol{E}^{H}:=$ $\left(E_{i j k l}^{H}\right)$ can be written as a symmetric and usually dense matrix. The computation of the homogenized elasticity coefficients can be done analytically for some specific geometries as, for instance, layered materials or checkerboard structures. In the case of more complicated microstructures, the computation of $E_{i j k l}^{H}$ has to be done numerically through a suitable microscopic modeling.

Once the constant homogenized coefficients from (3.9) have been computed, one comes up with the homogenized macroscopic equation (3.7) given in a weak form analogously to (2.6) as follows:

$$
\int_{\Omega} \boldsymbol{e}^{T}(\boldsymbol{v}) \boldsymbol{\sigma}\left(\boldsymbol{u}^{(0)}\right) d \Omega=\int_{\Omega} \boldsymbol{v}^{T} \boldsymbol{b} d \Omega+\int_{\Gamma_{T}} \boldsymbol{v}^{T} \boldsymbol{t} d \Gamma, \quad \forall \boldsymbol{v} \in U_{0}
$$

where $\boldsymbol{u}^{(0)}(\boldsymbol{x}):=\boldsymbol{u}^{(0)}(\boldsymbol{x}, \boldsymbol{y})$ is the homogenized solution, the leading term in (3.6).

Remark 3.1. The classical homogenization method as described above neither can be used for the suitable modeling of microstructural size effects nor does it appropriately reflect the physics in local regions of large gradients, since it relies on the assumption of uniformity of the macroscopic stress-strain fields. These drawbacks can be overcome by an extension of the classical homogenization method using a gradient-enhanced computational approach that leads to a higher-order macroscopic continuum (cf., e.g., [20] and the references therein). 


\section{Implementation of the multi-scale method}

In this section, we comment on the implementation of the proposed multi-scale method based on the asymptotic homogenization theory together with the mesh superposition method. The homogenization approach is used on the global domain excluding the vicinity of the crack where the periodicity of the microstructures is lost and this approach is not applicable. The crack is considered in the local domain (patch). Two independent (global and local) meshes are generated in the global and local domains, respectively, avoiding matching of the nodes in the two meshes. The patch is allowed to have an arbitrary geometry with respect to the underlying global finite elements. As explained in Section 2, the local mesh is superimposed on the global mesh in such a way that both meshes not necessarily coincide. The total displacement field from (2.9) is approximated by adding global (underlying) and local (superimposed) fields and hence it is discontinuous across the crack.

With the help of the homogenization technique discussed in Section 3 the homogenized macroscopic problem (3.7) defined in $\Omega$ is involved in the governing equation (2.1). Furthermore, introducing global and local meshes, the global displacement field $\boldsymbol{u}^{G}$ is expressed by the homogenized displacement $\boldsymbol{u}^{(0)}$, the leading term in the asymptotic expansion form (3.6). The homogenized elasticity problem in a weak form (3.10) is transformed to the solution of system (2.15) with a symmetric and, usually, sparse stiffness matrix coupling the integrands on the global and local meshes. Note that the elasticity (constitutive) tensor $\boldsymbol{E}^{G}$ in the expression (2.16) is replaced now by the homogenized elasticity tensor $\boldsymbol{E}^{H}$ with components computed by (3.9).

The multi-scale procedure is realized by the following algorithm.

\section{Multi-Scale Algorithm (MSA)}

Step 1. Select a unit microstructure $Y$ in the heterogeneous material.

Step 2. Solve the elasticity equation (3.8) to find the characteristic displacement fields $\boldsymbol{\xi}^{k l}$.

Step 3. Compute the homogenized elasticity coefficients by (3.9) and set $\boldsymbol{E}^{G}=\boldsymbol{E}^{H}$.

Step 4. Generate a global finite element mesh in $\Omega$ on the macroscopic homogenized model. Step 5. Introduce a local (discontinuous) mesh in the vicinity of the crack $\Gamma_{C}$.

Step 6. Solve (2.15) to find $\boldsymbol{u}^{G}$ and $\boldsymbol{u}^{L}$, the displacements on the global and local meshes. Step 7. Substitute $\boldsymbol{u}^{G}$ and $\boldsymbol{u}^{L}$ into (2.11) and (2.12) to find the strains and stresses.

From the algorithm above, one can see that three finite element meshes have to be generated on three different domains. First, Step 1 requires a finite element mesh in the unit microstructure. Due to the singularities around the pore located at the center of the microcell, adaptive mesh refinement is used based on reliable and efficient a posteriori error estimators. Numerical experiments are presented in the last section. Second, a global finite element mesh by a decomposition of $\Omega$ into triangles/tetrahedra is generated in Step 4 . Third, a local mesh has to be introduced in Step 5 in the vicinity of the crack. This mesh usually contains discontinuities. Recently, the XFEM has become a popular method for modeling cracks (see, e.g., $[22,24]$ and the references therein). The method is based on a standard displacement finite element approximation which is enriched near the crack by incorporating both discontinuous fields and near-tip asymptotic fields through a partition of unity property [23]. Details are given in the next Section 5 .

Another difficult problem that arises in the mesh superposition method is the numerical integration using Gauss quadratures. The problem appears due to the arbitrary patching of the local mesh incorporating a discontinuous field across the crack faces. The use of standard 
Gauss quadrature may not adequately integrate the discontinuous fields to represent the jump in displacement across the crack line. The modeling of discontinuous fields by means of the $s$-version of the FEM is discussed in [10]. For instance, the numerical integration of (2.17) requires that $\boldsymbol{B}^{G}(\boldsymbol{x})$ to be evaluated at the Gauss points in the local mesh. However, the Gauss quadrature points of the global and local meshes do not coincide due to the arbitrary geometry of the patch. Various approaches to the numerical integration of the weak form over the overlapped finite elements can be found in $[10,22,24,28,31]$.

\section{The extended finite element method}

The eXtended Finite Element Method (XFEM) [3,24] allows treating crack problems without meshing the discontinuity surface. This is possible through enrichment of the standard polynomial finite element space with special functions: discontinuous, to account for the displacement jump, and crack-tip fields to reduce the mesh density required for accurate fracture parameter computations. The method is now becoming quite mature, and has already been applied to industrial fracture mechanics problems (see, cf. [7] and the references therein).

Some finite elements are split by a crack and others contain crack tips. Denote by $\mathcal{N}_{c r}$ the set of nodes whose support is cut by a crack and by $\mathcal{N}_{t i p}$ the set of nodes whose support contains the crack tip. In Fig. 5.1 the circled nodes (set of nodes $\mathcal{N}_{c r}$ ) are enriched with the step function whereas the squared nodes (set of nodes $\mathcal{N}_{t i p}$ ) are enriched with the crack tip functions.
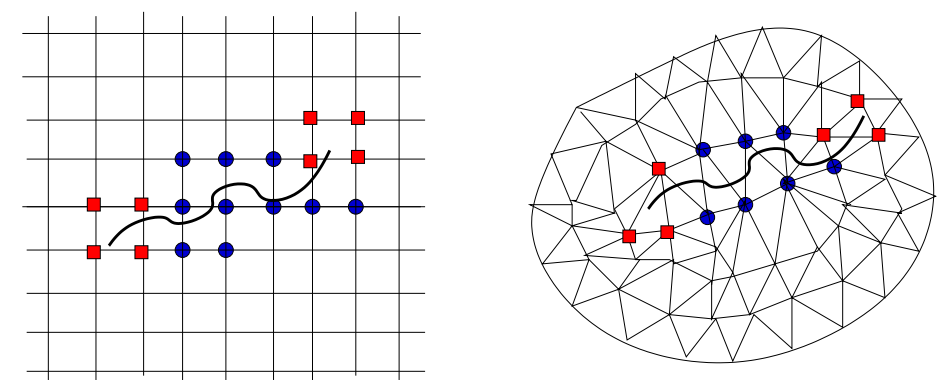

Fig. 5.1. Selection of enriched nodes on: a structured mesh (left); an unstructured mesh (right)

The XFEM approximation reads

$\boldsymbol{u}^{h}(\boldsymbol{x})=\sum_{I \in \mathcal{N}} N_{I}(\boldsymbol{x}) \boldsymbol{u}_{I}+\sum_{J \in \mathcal{N}_{c r}} \widetilde{N}_{J}(\boldsymbol{x})\left(H(\boldsymbol{x})-H\left(\boldsymbol{x}_{J}\right)\right) \boldsymbol{a}_{J}+\sum_{K \in \mathcal{N}_{t i p}} \widetilde{N}_{K}(\boldsymbol{x}) \sum_{\alpha=1}^{4}\left(C_{\alpha}(\boldsymbol{x})-C_{\alpha}\left(\boldsymbol{x}_{K}\right)\right) \boldsymbol{c}_{\alpha K}$,

where $\boldsymbol{u}_{I}$ are unknown discrete displacements, and $\boldsymbol{a}_{J}$ and $\boldsymbol{c}_{\alpha K}$ are enrichment nodal variables. Here, we consider the scalar finite element shape functions which, for convenience, are chosen equal with respect to each displacement component. Note that the shape functions $\widetilde{N}_{J}(\boldsymbol{x})$ associated with the enrichment can differ from the shape functions $N_{I}(\boldsymbol{x})$ used for the standard part of the displacement approximation. $H(\boldsymbol{x})$ is a modified Heaviside function which takes on the value +1 above the crack and -1 below the crack, i.e.,

$$
H(\boldsymbol{x})= \begin{cases}+1, & \text { if }\left(\boldsymbol{x}-\boldsymbol{x}^{*}\right) \cdot \boldsymbol{n} \geqslant 0 \\ -1, & \text { otherwise }\end{cases}
$$


where $\boldsymbol{x}$ is a sample point, $\boldsymbol{x}^{*}$ (lies on the crack) is the closest point projection of $\boldsymbol{x}$, and $\boldsymbol{n}$ is the unit outward normal to the crack at $\boldsymbol{x}^{*}$. The crack tip enrichment functions $C_{\alpha}(\boldsymbol{x})$, which span the near tip asymptotic field, are defined as

$$
\boldsymbol{C} \equiv\left[C_{1}, C_{2}, C_{3}, C_{4}\right]=\left[\sqrt{r} \sin \frac{\theta}{2}, \sqrt{r} \cos \frac{\theta}{2}, \sqrt{r} \sin \frac{\theta}{2} \cos \theta, \sqrt{r} \cos \frac{\theta}{2} \cos \theta\right] .
$$

Here, $r$ and $\theta$ are polar coordinates in the local crack tip coordinate system. Note that the first function $\sqrt{r} \sin (\theta / 2)$ in (5.2) is discontinuous across the crack and thus represents the discontinuity near the crack (see, cf. [24]). The remaining three functions in (5.2) are continuous. They are added to get accurate results on relatively coarse meshes.

From the enriched approximation (5.1) the Bubnov - Galerkin procedure gives discrete equations of the form $\boldsymbol{K} \boldsymbol{u}=\boldsymbol{f}$ with a stiffness matrix $\boldsymbol{K}$ and a force vector $\boldsymbol{f}$. Numerical integration for split elements is done here by partitioning the elements into subtriangles within the Delaunay triangulation. For each subtriangle high-order Gauss quadrature rule is used. Interested readers can refer to [7] for details.

\section{Numerical experiments}

Preforms of natural materials like wood permit the manufacturing cellular ceramics with unidirectional porous structures. The natural wood morphologies are characterized by an open porous system of tracheidal cells providing transportation paths for water and minerals in living plants (cf., e.g., [26]). The inherent cellular highly open porous system, accessible for infiltration of various liquid or gaseous metals is used to design novel porous ceramics. The transformation of carbonized wood into porous carbide ceramics can be done by infiltrationreaction processes with various carbide forming metals (e.g., $\mathrm{Si}, \mathrm{Ti}$ ).

In recent years, a great deal of research has been focused on the production of silicon carbide $(\mathrm{SiC})$-based biomorphic microcellular ceramics. For details of the processing scheme and mechanical properties, we refer the reader to [14]. The production process relies on advanced biotemplating methods and comprises several processing steps ranging from the preparation of appropriate carbonized preforms using high-temperature pyrolysis via chemical reactions by liquid- or gaseous-phase infiltrants to postprocessing such as cutting and etching. After the first step of processing, the total porosity of the resulting graphitelike carbon preform is approximately $25 \%$ less than the initial porosity of the dried wood and features a multimodal pore size distribution with pores of a diameter between $1 \mu \mathrm{m}$ to $200 \mu \mathrm{m}$. However, despite the shrinkage, the porous microstructure of the carbonized wood is retained after high-temperature pyrolysis (see Fig. 6.1 in the case of a pyrolyzed pine specimen for both Si-gas and Si-polymer infiltration). We note that different kinds of wood exhibit different microstructures.
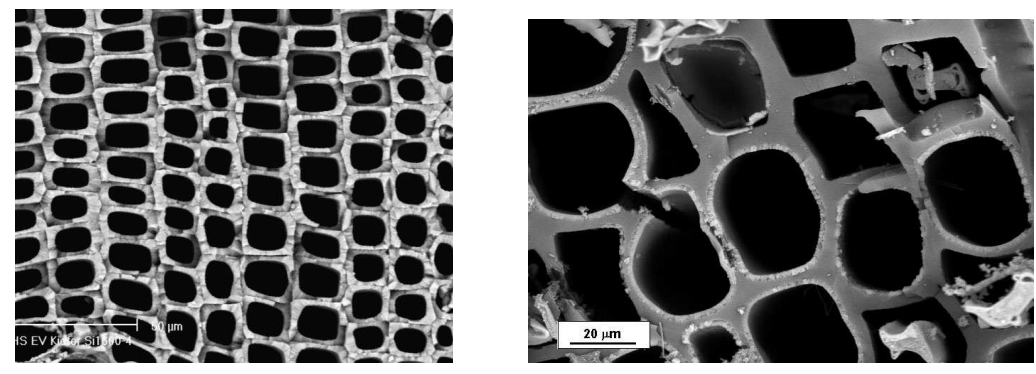

F i g. 6.1. SiC ceramics derived from pine wood: Si-gas infiltration (left); Si-polymer infiltration (right) 
The new bioorganic ceramic materials aroused considerable interest in many research fields, primarily in materials science and engineering. They have found a lot of practical applications as filters and catalysts in chemical processing, heat exchangers, and thermal insulation devices.

In Step 1 of the algorithm MSA we consider a stationary microstructure with a geometrically simple tracheidal periodicity cell $Y=[0,1]^{d}, d=2,3$, (see Fig. 6.2) consisting of an outer layer of carbon $(\mathrm{C})$, an inner layer of silicon carbide $(\mathrm{SiC})$, and a centered pore channel ( $\mathrm{P}$, no material). A two-dimensional unit periodicity cell for $\mathrm{SiC}$ ceramics is presented in Fig. 6.2,b. One can also deal with the so-called pure $\mathrm{SiC}$ ceramics when enough silicon is infiltrated in the pore channel as a result of the reaction between carbon and silicon (see Fig. 6.2, $c$ for $d=2$ ). Assume that the macroscopic material is constructed by introducing an infimum of periodically distributed infinitesimal microstructures. For simplicity, we suppose homogeneous and isotropic constituents in terms of carbon and SiC. The Young modulus $E$ (in $\mathrm{GPa}$ ) and the Poisson ratio $\nu$ of our two materials are, respectively, $E=10, \nu=0.22$ for carbon and $E=410, \nu=0.14$ for $\mathrm{SiC}$.

a)

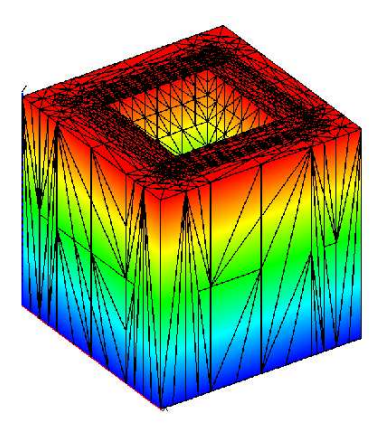

b)

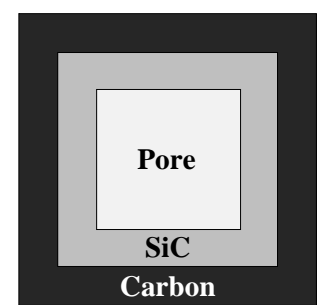

c)

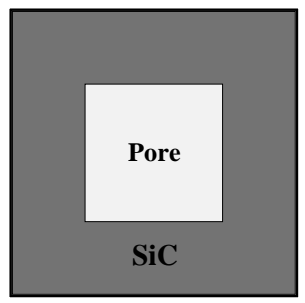

Fig. 6.2. 3-D unit periodicity cell $Y(a), 2$-D unit periodicity cell $Y=$ $\mathrm{P} \cup \mathrm{SiC} \cup \mathrm{C}(\mathrm{SiC}$ ceramics) (b), 2-D unit periodicity cell $Y=\mathrm{P} \cup \mathrm{SiC}$ (pure $\mathrm{SiC}$ ceramics) $(c)$

The computation of the characteristic displacement fields $\boldsymbol{\xi}^{k l}$ (Step 2) and the homogenized elasticity coefficients (Step 3) requires the solution of linear elastic boundary value problems with the periodicity cell $Y$ as the computational domain. Due to the composite character of the cell there are material interfaces where the solution changes significantly. Hence, local refinement of the underlying finite element mesh is strongly advised.

We use an adaptive grid refinement strategy based on a posteriori error estimator of the Zienkiewicz - Zhu type [32] obtained by local averaging of the computed stress tensor. In particular, in the case of conforming P1 finite elements with respect to a simplicial triangulation $\mathcal{T}_{h}$ of $Y$ that aligns with the multilayer structure, the computed stress $\hat{\boldsymbol{\sigma}}$ is discontinuous across the interior element boundaries. We compute a continuous recovered stress $\boldsymbol{\sigma}^{*}$ with continuous, piecewise linear components as follows: for a node $P$, we compute $\boldsymbol{\sigma}^{*}(P)$ by averaging the stresses over the elements that share $P$ as a common vertex. We use the $L^{2}$-norm $\eta_{T}:=\left\|\boldsymbol{\sigma}^{*}-\hat{\boldsymbol{\sigma}}\right\|_{0, T}, T \in \mathcal{T}_{h}$, as a local recovery estimator and obtain a refine triangulation by using the longest edge bisection for those elements $T \in \mathcal{T}_{h}$ for which $\eta_{T} \geqslant\left(\max _{T^{\prime} \in \mathcal{T}_{h}} \eta_{T^{\prime}}\right) / 2$ (for further details see [17]). Hence, the adaptivity procedure is local and computationally cheap. In [15], we presented some numerical experiments on a plane microstructure by using an adaptive mesh-refinement technique for the computation of recovered stresses. In the case of 3-d implementations, we decompose the periodic microcell $Y$ first in hexahedra 
and further we use continuous, piecewise linear finite elements on tetrahedral shape regular meshes, as shown in Fig. 6.3. Due to the symmetry of the periodic displacements $\boldsymbol{\xi}^{k l}=\boldsymbol{\xi}^{l k}$, Eq. (3.8) in Step 2 is computed numerically 3 times in the case $d=2$ and, accordingly, 6 times in the case $d=3$.

The discrete elasticity problem in the microstructure is solved iteratively by using the Preconditioned Conjugate Gradient (PCG) method with Incomplete Cholesky (IC) or Algebraic MultiGrid method (AMG) as a preconditioner. Various approaches to the construction of a preconditioner for the stiffness matrix and numerous computational results for the convergence history of both preconditioners are discussed in [16].
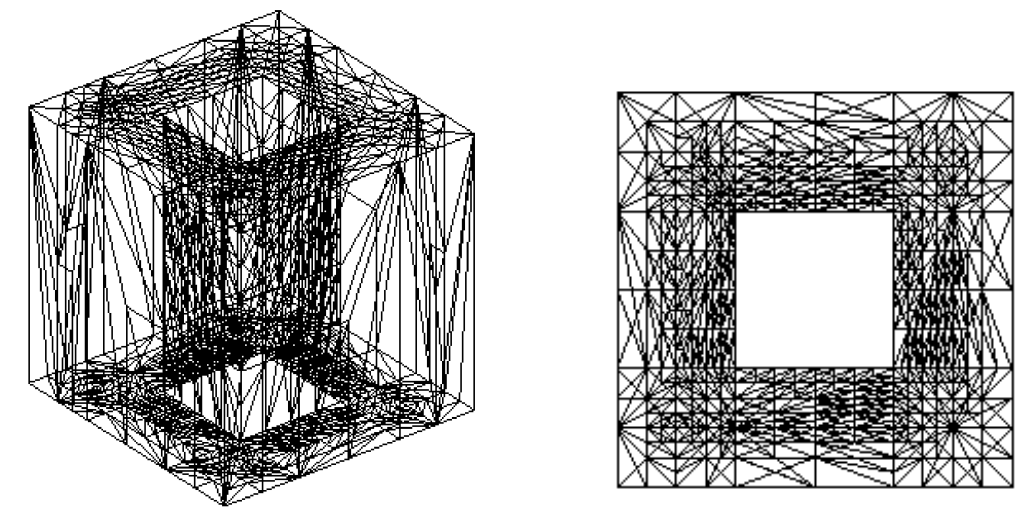

Fig. 6.3. Adaptive refinement of 3-D unit periodicity cell $Y$ (left), cross section of $Y$ (right)

Denote the global density of the solid material part in the microstructure by $\mu, 0<$ $\mu<1$. If $\mu$ is relatively small, we speak of early wood (grown in spring and summer) and, accordingly, of late wood (grown in autumn and winter) for values of $\mu$, close to 1 . In Table 6.1 we report some values of the computed 3-dimensional homogenized coefficients with respect to the adaptive refinement level for early wood with a density of $36 \%$ and late wood with a density of $84 \%$.

Table 6.1. Homogenized elasticity coefficients

\begin{tabular}{|c|c|c|c|c|c|c|c|c|c|c|c|c|}
\hline & \multicolumn{6}{|c|}{ Early wood, density $\mu=36 \%$} & \multicolumn{5}{|c|}{ Late wood, density $\mu=84 \%$} \\
\cline { 2 - 13 } Level & $E_{1111}^{H}$ & $E_{2222}^{H}$ & $E_{3333}^{H}$ & $E_{1212}^{H}$ & $E_{2323}^{H}$ & $E_{1313}^{H}$ & $E_{1111}^{H}$ & $E_{2222}^{H}$ & $E_{3333}^{H}$ & $E_{1212}^{H}$ & $E_{2323}^{H}$ & $E_{1313}^{H}$ \\
\hline 1 & 176.1 & 188.6 & 215.9 & 64.9 & 73.6 & 63.0 & 167.8 & 174.0 & 180.1 & 62.7 & 66.5 & 61.8 \\
2 & 193.8 & 223.4 & 232.7 & 78.7 & 96.3 & 75.9 & 179.6 & 193.4 & 195.8 & 75.5 & 80.0 & 71.4 \\
3 & 174.5 & 185.4 & 224.0 & 59.6 & 71.7 & 66.5 & 167.8 & 180.5 & 187.4 & 63.5 & 70.8 & 68.8 \\
4 & 190.0 & 171.7 & 234.0 & 69.8 & 78.8 & 79.4 & 171.9 & 158.1 & 193.9 & 53.7 & 60.0 & 70.9 \\
5 & 190.5 & 174.0 & 230.6 & 43.4 & 69.8 & 61.8 & 154.6 & 155.3 & 193.0 & 50.3 & 63.7 & 62.6 \\
6 & 155.7 & 166.9 & 225.9 & 38.8 & 68.1 & 59.1 & 154.3 & 127.0 & 190.6 & 38.0 & 51.1 & 49.7 \\
7 & 185.7 & 166.5 & 235.0 & 49.1 & 58.8 & 58.9 & 120.4 & 110.6 & 190.8 & 37.2 & 46.1 & 49.3 \\
8 & 157.8 & 147.1 & 231.1 & 39.6 & 60.8 & 57.0 & 105.4 & 102.8 & 189.7 & 31.1 & 46.1 & 47.3 \\
9 & 142.2 & 144.2 & 225.3 & 29.3 & 58.7 & 53.6 & 96.1 & 91.1 & 189.0 & 27.1 & 43.8 & 45.0 \\
10 & 132.6 & 140.9 & 227.1 & 27.9 & 55.3 & 56.0 & 89.0 & 87.5 & 188.1 & 26.3 & 43.6 & 43.7 \\
\hline
\end{tabular}

Our experience from [16] shows that the AMG-preconditioner has a much better efficiency compared to the IC-preconditioner as to both the number of iterations and the CPU-time. 
Some convergence results for various values of the density and more details about the discretization parameters on successive adaptive refinement levels are presented in Table 6.2. Here, we have denoted by NT the number of tetrahedra, by NN the number of nodes, by NDOF the number of degrees of freedom after eliminating the points with prescribed displacements, by ITER the number of iterations by the PCG method, and by CPU the time in seconds for the iterative solver using the AMG-preconditioner.

Table 6.2. Convergence results with the AMG preconditioner

\begin{tabular}{|c|c|c|c|c|c|c|c|c|}
\hline Density & Level & 4 & 5 & 6 & 7 & 8 & 9 & 10 \\
\hline$\mu=19 \%$ & NT & 613 & 968 & 1595 & 2175 & 3187 & 3546 & 7262 \\
& NN & 217 & 294 & 428 & 549 & 764 & 844 & 1623 \\
& NDOF & 207 & 354 & 588 & 846 & 1314 & 1533 & 3417 \\
& ITER & 15 & 19 & 24 & 42 & 50 & 53 & 93 \\
& CPU & 0.1 & 0.2 & 0.5 & 0.9 & 1.7 & 2.1 & 7.4 \\
\hline \multirow{2}{*}{$\mu=91 \%$} & NT & 823 & 1257 & 2122 & 4196 & 7046 & 12190 & 20822 \\
& NN & 266 & 365 & 552 & 984 & 1660 & 2660 & 4267 \\
& NDOF & 276 & 486 & 792 & 1740 & 3369 & 5667 & 9528 \\
& ITER & 13 & 17 & 24 & 31 & 43 & 49 & 59 \\
& CPU & 0.1 & 0.4 & 0.7 & 2.1 & 4.8 & 10.1 & 21.7 \\
\hline
\end{tabular}

The failure of biotemplated microcellular $\mathrm{SiC}$ ceramics from wood by the formation and propagation of cracks has been investigated experimentally in [14]. The failure is due to the higher sensitivity of the bending strength (compared to the elasticity) on the microstructural anisotropy and depends on the loading conditions. During the uniaxial compression testing, the specimen was deformed in a linear elastic mode up to a critical stress where the crack growth started (see Fig. 5.1(right)). In the case of two different loading orientations parallel to the axial direction, large unfilled tracheidal pores are oriented perpendicular to the normal tensile stress and are usually zones of crack initiation as soon as a critical load is reached. The crack propagates in planes with a high pore density parallel to the pore channel orientation.

For the local critical region containing a crack, the approximation of the displacement field has to be enhanced by additional shape functions taking into account the discontinuities across the crack. We rely on the XFEM introducing specific discontinuous displacement functions defining the crack and the singularities at the crack-tips. Computations of the fracture mechanics are performed using the XFEM library [7]. As a first attempt, we consider a crack in the macroscopic homogenized model. Table 6.3 presents the evaluated data for the homogenized Young modulus $E^{H}$ (in GPa) and the homogenized Poisson ratio $\nu^{H}$ in the case of pure $\mathrm{SiC}$ ceramics. The density of the $\mathrm{SiC}$ layer is denoted by $\mu_{S i C}$ and thus the porosity is determined by meas $(Y)-\mu_{S i C}$. As would be expected, $E^{H}$ and $\nu^{H}$ decrease with increasing porosity, i.e., the mechanical and elasticity properties of the ceramic materials are strongly influenced by the porosity and decrease when it becomes higher.

In all problems, for both crack tips we define the energy release rate as

$$
G=\frac{1}{E^{\prime}}\left(K_{I}^{2}+K_{I I}^{2}\right) \quad \text { with } \quad E^{\prime}=\frac{E^{H}}{1-\left(\nu^{H}\right)^{2}}
$$

in the case of plane strains. In our experiments, the material characteristics $E^{H}$ and $\nu^{H}$ in the macroscopic homogenized model are computed preliminarily. During the brittle fracture in the obtained homogenized material, the energy release rate remains a constant depending only on the material. In general, the fracture energy depends on the microstructure and 
should be obtained by taking into account the microscopic characteristics as an additional step.

The stress intensity factors (SIFs), $K_{I}$ and $K_{I I}$, for modes I and II, respectively, are determined using the domain form of the interaction integrals [25]. We are interested in the effect of porosity on the energy release rate. Consider a center crack in an infinite plate under remote, unit, uniaxial tension. The geometry of the plate is $(2 \times 6)$, the crack length is 0.25 , and the mesh, completely regular and non-conforming to the crack, contains 2701 four-node quadrilateral elements. The radius of the circular interaction integral domain is twice the size of the element containing the tip. Note that in this case, the exact SIFs are actually known analytically. The computed SIFs are within one percent of the exact values.

\section{Table 6.3. Homogenized material con- stants for pure $\mathrm{SiC}$ ceramics}

\begin{tabular}{|c|c|c|c|}
\hline Porosity & $\mu_{\mathrm{SiC}}$ & $E^{H}(\mathrm{GPa})$ & $\nu^{H}$ \\
\hline 0.9025 & 0.0975 & 216.10 & 0.016 \\
0.7225 & 0.2775 & 231.94 & 0.040 \\
0.5625 & 0.4375 & 248.93 & 0.062 \\
0.4225 & 0.5775 & 267.94 & 0.083 \\
0.3025 & 0.6975 & 289.27 & 0.104 \\
0.2025 & 0.7975 & 314.16 & 0.124 \\
0.1225 & 0.8775 & 342.27 & 0.138 \\
0.0025 & 0.9975 & 409.14 & 0.139 \\
\hline
\end{tabular}

The evolution of the energy release rate $G$ as a function of porosity in the case of pure $\mathrm{SiC}$ ceramics is given in Fig. 6.4. One can observe that for relatively dense materials, with a porosity close to 0 , the energy release rate $G$ remains at the same level and increases slightly (by an order of magnitude $10^{-4}$ ) with increasing porosity, i.e., with increasing brittleness of the material.

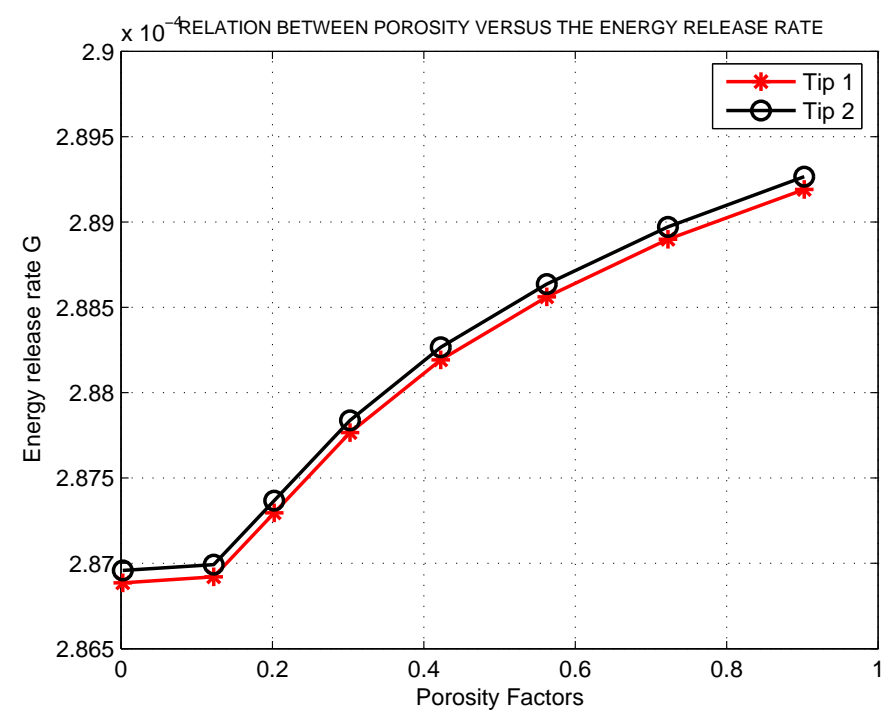

Fig. 6.4. Porosity versus the energy release rate $G$ for pure $\mathrm{SiC}$ ceramics

\section{Conclusions}

We have considered the failure of microstructural materials due to the initiation and propagation of cracks in places of a high porosity in the microstructure. For such materials, the 
asymptotic homogenization theory cannot be applied in a standard way, since in the vicinity of the crack the microscopic periodicity is lost. The modeling of the crack problem relies on a combination of the homogenization approach for the macroscopic model and the mesh superposition method, also known as the $s$-version of the FEM. The latter method relies on the simultaneous use of independent (global and local) meshes and a superposition technique in the critical region near the crack. The global mesh covers the entire domain where the homogenized macroscopic material model is considered. The local mesh in the vicinity of the crack uses discontinuous shape functions and is arbitrarily superimposed on the global continuous mesh. A multi-scale algorithm is proposed for the solution of the crack problem. Numerical experiments with the use of the XFEM in preliminary homogenized brittle ceramic materials are discussed. A brief description of the XFEM enriched with additional functions by partitioning the unity property is presented.

Acknowledgments. The authors express their thanks to the anonymous referee for various helpful comments and suggestions that led to an improvement of the manuscript. They also gratefully acknowledge the several fruitful discussions with Dr. S. Bordas related to the XFEM library [7].

The work was sponsored in part by the German NSF Priority Program (DFG SPP) 1253 under Grant HO877 9-2. The research of the first author was supported by NSF grants DMS-0511624, DMS-0707602, DMS-0810176, DMS-0811153, and DMS-0914788. The second author has been further supported in part by the Project (Account number 03000481), FSP AMMO at the University of Applied Sciences, Bielefeld, Germany, and by the Bulgarian NSF Grant DO 02-115/08.

\section{References}

1. G. Allaire, Shape Optimization by the Homogenization Method, Springer, New York, 2002.

2. N. Bakhvalov qnd G. Panasenko, Averaging Processes in Periodic Media, Nauka, Moscow, 1984.

3. T. Belytschko, N. Moës, S. Usui, and C. Parimi, Arbitrary discontinuities in finite elements, Int. J. Numer. Methods Eng. 50 (2001), no. 4, pp. 993-1013.

4. M. P. Bendsøe and O. Sigmund, Topology Optimization: Theory, Methods and Applications, Springer, Berlin-Heidelberg-New York, 2003.

5. A. Bensoussan, J. L. Lions, and G. Papanicolaou, Asymptotic Analysis for Periodic Structures, NorthHolland, Elsevier Science Publishers, Amsterdam, 1978.

6. S. Bordas, R. H.W. Hoppe, and S.I. Petrova, Mechanical failure in microstructural heterogeneous materials, Proc. NM\&A’06, August 20-24, 2006, Borovets, Bulgaria, In: Lect. Notes Comput. Sci. (T. Boyanov et al., eds.), Springer, 4310 (2007), pp. 533-541.

7. S. Bordas, V.P. Nguyen, C. Dunant, A. Guidoum, and H. Nguyen-Dang, An extended finite element library, Int. J. Numer. Methods Eng., 71 (2007), no. 6, pp. 703-732.

8. W. E. B. Engquist, The heterogeneous multiscale methods, Comm. Math. Sci. 1 (2003), no. 1, pp. 87132 .

9. J. Fish, The s-version of the finite element method, Comput. Struct. 43 (1992), no. 3, pp. 539-547.

10. J. Fish, Hierarchical modelling of discontinuous fields, Commun. Appl. Numer. Methods. 8 (1992), no. 7 , pp. $443-453$.

11. J. Fish, S. Markolefas, R. Guttal, and P. Nayak, On adaptive multilevel superposition of finite element meshes for linear elastostatics, Appl. Numer. Math. 14 (1994), no.1-3, pp. 135-164.

12. L. J. Gibson and M. F. Ashby, Cellular Solids, Structure, and Properties, Pergamon Press, New York, 1988.

13. J.Fish and R. Guttal, The $s$-version of finite element method for laminated composites, Int. J. Numer. Methods Eng. 39 (1996), no. 22, pp. 3641-3662.

14. P. Greil, T. Lifka, and A. Kaindl. Biomorphic cellular silicon carbide ceramics from wood: I. Processing and microstructure, and II. Mechanical properties, J. Europ. Cer. Soc. 18 (1998), pp. 1961-1973 
and pp. $1975-1983$.

15. R. H. W. Hoppe and S. I. Petrova, Optimal shape design in biomimetics based on homogenization and adaptivity, Math. Comput. Simul. 65 (2004), no. (3), pp. 257-272.

16. R.H.W. Hoppe and S. I. Petrova, Shape optimization of biomorphic ceramics with microstructures by homogenization modeling, Analysis, Modeling and Simulation of Multiscale Problems (A. Mielke, ed.), Springer, 2006, pp. 395-424.

17. R. H. W. Hoppe, S. I. Petrova, and Y. Vassilevski, Adaptive grid refinement for computation of the homogenized elasticity tensor, Lect. Notes Comput. Sci., Vol. 2907 (I. Lirkov et al., eds.), Springer, 2004, pp. 371-378.

18. V. V. Jikov, S. M. Kozlov, and O. A. Oleinik, Homogenization of Differential Operators and Integral Functionals, Springer, 1994.

19. L. M. Kachanov, Introduction to Continuum Damage Mechanics, Kluwer Academic Publishers, Dordrecht, 1986 .

20. V. Kouznetsova, M. G. D. Geers, and W. A. M. Brekelmans, Multi-scale constitutive modelling of heterogeneous materials with a gradient-enhanced computational homogenization scheme, Int. J. Numer. Methods Eng. 52 (2002), pp. 1235-1260.

21. K. Lee, S. Moorthy, and S. Ghosh, Multiple scale computational model for damage in composite materials, Comput. Methods Appl. Mech. Engrg. 172 (1999), no. 1-4, pp. 175-201.

22. S.-H. Lee, J.-H. Song, Y.-C. Yoon, G. Zi, and T. Belytschko, Combined extended and superimposed finite element method for cracks, Int. J. Numer. Methods Eng. 59 (2004), no. 8, pp. 1119-1136.

23. J. Melenk and I. Babuska, The partition of unity finite element method: Basic theory and applications, Comput. Methods Appl. Mech. Eng. 139 (1996), no. 1-4, pp. 289-314.

24. N. Moës, J. Dolbow, and T. Belytschko, A finite element method for crack growth without remeshing, Int. J. Numer. Methods Eng. 46 (1999), no. 1, pp. 131-150.

25. B. Moran and C.F. Shih, Crack tip and associated domain integrals from momentum and energy balance, Eng. Fract. Mech., 27 (1987), pp. 615-641.

26. T. Ota, M. Takahashi, T. Hibi, M. Ozawa, S. Suzuki, Y. Hikichi, and H. Suzuki, Biomimetic process for producing SiC wood, J. Amer. Ceram. Soc. 78 (1995), pp. 3409-3411.

27. H. Rafii-Tabar, L. Hua, and M. Cross, A multi-scale atomistic-continuum modelling of crack propagation in a two-dimensional macroscopic plate, J. Phys.: Condens. Matter. 10 (1998), pp. 2375-2387.

28. M. Rashid, The arbitrary local mesh replacement method: An alternative to remeshing for crack propagation analysis, Comput. Methods Appl. Mech. Eng. 154 (1998), no.1-2, pp. 133-150.

29. E. Sanchez-Palencia, Non-homogeneous Media and Vibration Theory, Lecture Notes in Physics, 127, Springer, Berlin-Heidelberg, 1980.

30. R. N.L.Smith and D. Della-Ventura, A superposition method for the solution of crack problems, Commun. Numer. Methods Eng. 11 (1995), no. 3, pp. 243-254.

31. N. Takano, M.Zako, and Y. Okuno, Multi-scale finite element analysis of porous materials and components by asymptotic homogenization theory and enhanced mesh superposition method, Modelling Simul. Mater. Sci. Eng. 11 (2003), pp. 137-156.

32. O. C. Zienkiewicz and J.Z.Zhu, A simple error estimator and adaptive procedure for practical engineering analysis, Intern. J. Numer. Methods Eng. 24 (1987), pp. 337-357. 